\title{
Monitoring Plasmodium vivax chloroquine sensitivity along China-Myanmar border of Yunnan Province, China during 2008-2013
}

Hui Liu', Heng-lin Yang ${ }^{1 *}$, Lin-hua Tang ${ }^{2}$, Xing-liang Li ${ }^{1}$, Fang Huang ${ }^{2}$, Jia-zhi Wang ${ }^{3}$, Chun-fu Li ${ }^{1}$, Heng-ye Wang ${ }^{1}$, Ren-hua Nie ${ }^{1}$, Xiang-rui Guo ${ }^{4}$, Ying-xue Lin ${ }^{4}$, Mei Li ${ }^{2}$ and Jian-Wei Xu ${ }^{1 *}$

\begin{abstract}
Background: Plasmodium vivax is the most widespread of the malaria parasites infecting human hosts. In malaria-eliminating settings, both imported and local malaria predominantly occurs in border areas, and most of them are $P$. vivax. Chloroquine (CQ) is the first-line drug for $P$. vivax treatment in China. To understand CQ sensitivity in $P$. vivax, in vivo monitoring of CQ resistance was conducted along the China-Myanmar border from 2008 to 2013.

Methods: Eligible patients with mono-infections of $P$. vivax were recruited to this study after obtaining full informed consent. CQ tablets for different categories of kg body weight ranges were given once a day for three days. Patients were followed up for 28 days. PCR was conducted to distinguish between re-infection and recrudescence, to confirm the Plasmodium species. The data were entered and analysed by the Kaplan-Meier method. Treatment outcome and sensitivity were classified according to the WHO recommended standards.
\end{abstract}

Results: 603 patients were completed valid follow-up. The fever clearance time and asexual parasite clearance times were, respectively, $22.2 \pm 10.2$ and $38.1 \pm 12.6$ hours. 594 (98.5\%) patients were adequate clinical and parasitological response (ACPR), and nine (1.5\%) patients, who were late clinical failure (LCF) or resistant response level I (RI), were imported from the neighbouring districts of Myanmar.

Conclusion: In terms of efficacy, CQ is still effective for vivax malaria treatment. Plasmodium vivax CQ sensitivity had not significantly changed along the China-Myanmar border of Yunnan Province, China.

Keywords: Plasmodium vivax, Chloroquine, Resistance, China-Myanmar border

\section{Background}

Malaria is still a global public health problem. The World Health Organization (WHO) estimated that between 2000 and 2010 , global malaria incidence decreased by $17 \%$ and malaria-specific mortality rates decreased by $26 \%$. Reported malaria cases have reduced by more than $50 \%$ in 34 of the 99 malaria-endemic countries [1]. China has declared a national policy for malaria elimination by 2020 $[2,3]$. In eliminating settings, malaria predominantly occurs in border areas, and imported cases tend to make up the majority of recorded cases caused by Plasmodium vivax [4]. Resistance to anti-malarial drugs has often threatened malaria elimination efforts and historically has

\footnotetext{
*Correspondence: yang_henglin@sohu.com; xjw426@163.com

'Yunnan Institute of Parasitic Diseases, Puer 665000, China

Full list of author information is available at the end of the article
}

led to the short-term resurgence of malaria incidence and deaths [5]. This can jeopardize progress and investment in combating malaria [6]. Plasmodium vivax is the most widespread of the malaria parasites infecting human hosts. Although there were an estimated 72-80 million $P$. vivax infections each year, it has not received as much public and scientific attention as Plasmodium falciparum [7]. In fact, $P$. vivax can lead to a disabling disease that can be fatal, and exacts a similar economic burden as falciparum malaria [8]. Finding and treating the P. vivax infection to prevent onward transmission is the determinant intervention. Chloroquine (CQ)-resistant $P$. vivax was first described in 1989 in Papua New Guinea [9], and the decline in the efficacy of CQ has been reported in some geographical sites [10]. CQ is the first-line drug for P. vivax 
treatment in China. Understanding P. vivax sensitivity to CQ is the basis of vivax malaria treatment with CQ. Knowledge of drug sensitivity is also needed to guide the local drug policy, so WHO strongly encourages countries in which $P$. vivax is endemic to carry out pilot studies to monitor the efficacy of CQ [11]. From 2008 to 2013, in vivo monitoring of $\mathrm{CQ}$ resistance in $P$. vivax was conducted to determine the dynamics of $P$. vivax sensitivity to $\mathrm{CQ}$ in the field along the China-Myanmar border.

\section{Methods}

\section{Surveillance sites and time}

Surveillance was carried out in Tengchong and Yangjiang, two counties on the China-Myanmar border (Figure 1). One of the main reasons for this selection of surveillance sites was that suitable vivax malaria patients were difficult to find in other parts of China. Most of the malaria cases in the two counties were imported from neighbouring districts of Myanmar. The surveillance activities were conducted in Tengchong County in 2008 and 2009, and expanded to Yingjiang County in 2010 and 2011, and only carried out in Yingjiang County because of so few malaria cases in Tengchong in 2012 and 2013.

\section{Patients and recruiting criteria}

Patients whose axillary temperature was $\geq 37.5^{\circ} \mathrm{C}$ or history of fever during the previous 24 hours were diagnosed based on microscopy of thick and thin blood smears. Patients with mono-infections of $P$. vivax were recruited to this study after obtaining full informed consent. Only patients older than one year presenting with parasite density $500-120,000$ parasites per $\mu \mathrm{L}$ were enrolled into the study. Imported malaria was identified as patients who had traveled from endemic areas of Myanmar within one month and were diagnosed as malaria in China [3]. Patients were excluded from the study if any of the following criteria were present: (1) positive pregnancy test or breastfeeding; (2) complicated malaria; (3) having taken any anti-malarial, sulpha, tetracycline and sulphone drugs within the previous seven days; (4) history of hypersensitivity to any of the study drugs; (5) severe dysfunction with kidney, liver and heart; (6) over 60 years old; and, (7) unable to follow up.

\section{Drug and administration}

CQ (GYZZ H31020423, 155 mg base per tablet) was made by Shanghai Sino-West Pharmaceutical Corp, China, and provided by The West Pacific Office of World Health

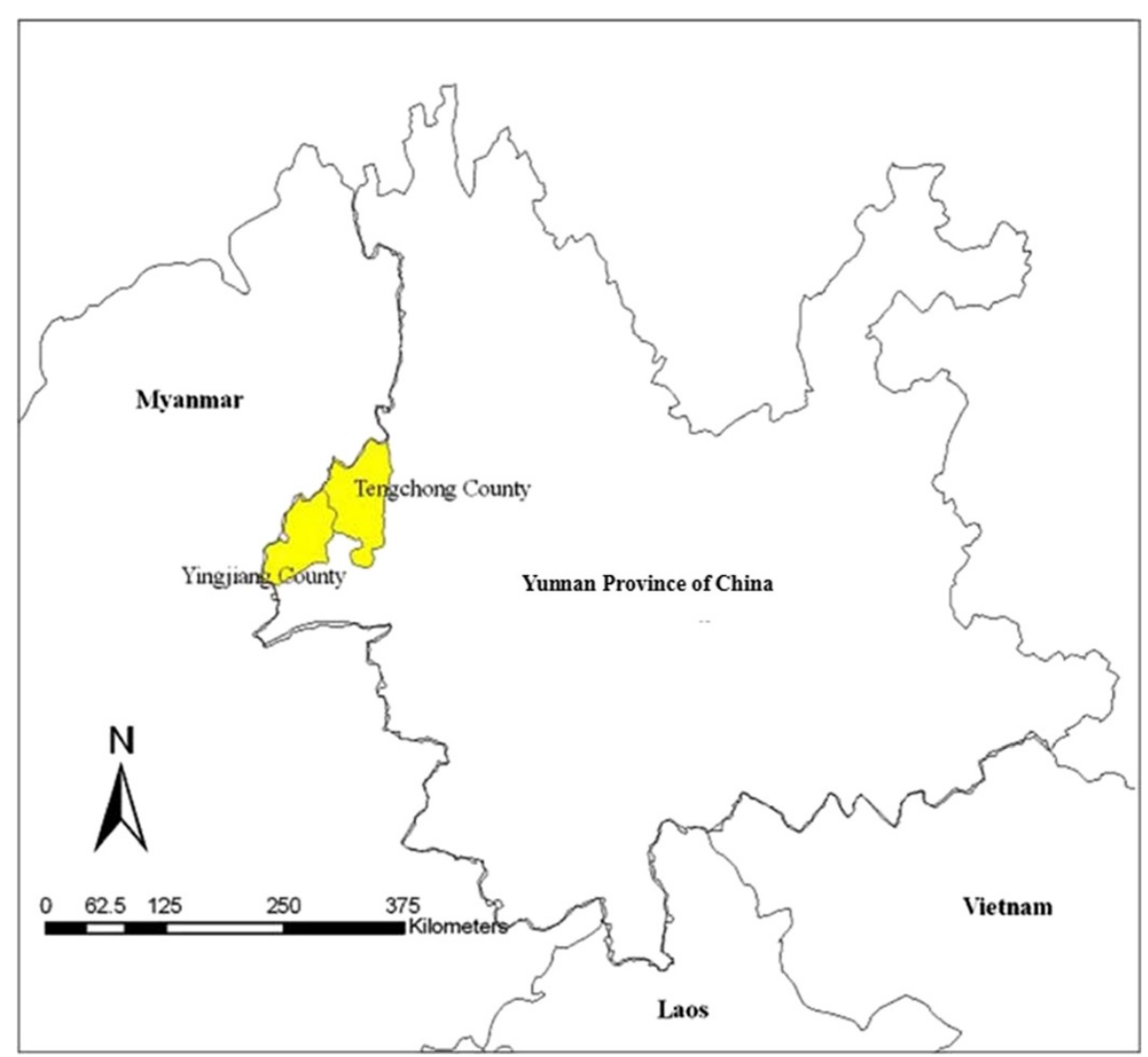

Figure 1 The location of Tengchong and Yingjiang counties relative to neighbouring countries. 
Organization (WPRO/WHO). CQ was given once a day for three days. The doses were recommended by WPRO/WHO too. For convenient administration of the drugs, the doses were calculated into tablets for different categories of $\mathrm{kg}$ body weight ranges (Table 1) [12]. Based on the recommendation of the China Ministry of Health, a standard adult ( $\geq 16$ years) dosage of primaquine tablets $(22.5 \mathrm{mg} /$ day $)$ was given for treatment of liver-stage parasites over eight days after completion of 28-day follow-up. The age based dosing was used for children, 3.75-5.625 mg/day for age 1-3 years, $7.5 \mathrm{mg} /$ day for age 4-6 years, $11.25 \mathrm{mg} /$ day for age $7-12$ years and $16.875 \mathrm{mg} /$ day for age 13-15 years over eight days [12].

\section{Laboratory and in vivo clinical monitoring}

Parasite microscopy was conducted on admission and every 12 hours while the patients stayed in hospital over the following seven days. Then patients were discharged from hospital and further parasitological examinations were performed on days 14, 21 and 28. Malaria blood films were stained with Giemsa and parasites were counted per 500 white blood cells. The number of parasites was calculated as per ul of blood by the level of 8,000 of leukocyte per ul [13]. A filter-paper dried blood spot (about 100 ul blood) was prepared on admission, days $3,7,14,21$, and 28 for polymerase chain reaction (PCR) to distinguish between re-infection and recrudescence, to confirm the Plasmodium species or to detect mixed infection. Improved Chelex 100 ion exchange method was used to extract DNA from blood filter paper samples, nested PCR and allele specific PCR techniques, agarose gel electrophoresis analysis and dot/southern blotting probe hybridization were employed for amplification, resolution and identification of the circumsporozoite protein (CSP) gene to distinguish reinfection from recrudescence or relapse. The primary PCR primers were 5' - TCCCCACGCACTGCAAAC ACAAT-3' (F) and 5' - CCGCAGGAGGTGCCACGTATAATT-3'(R). The secondary PCR primers were $5^{\prime}$ - GAAAATAAGCTGAA ACAAC-3' (F) and 5'-TACGTCACATTGGAC ACCT-3' (R) [14]. In case of failure after day 7, patients whose PCR results were unknown would be excluded from the analysis

Table 1 Number of chloroquine tablets (155 mg base per tablet) for each category of body weight range

\begin{tabular}{lllll}
\hline $\begin{array}{l}\text { Body weight } \\
\text { range }(\mathbf{k g})\end{array}$ & $\begin{array}{l}\text { Number } \\
\text { of tablets } \\
\text { Day 1 }\end{array}$ & $\begin{array}{l}\text { Number } \\
\text { of tablets } \\
\text { Day 2 }\end{array}$ & $\begin{array}{l}\text { Number } \\
\text { of tablets } \\
\text { Day 3 }\end{array}$ & $\begin{array}{l}\text { Total } \\
\text { tablets }\end{array}$ \\
\hline$\leq 12$ & 1 & 0.5 & 0.5 & 2 \\
$13-24$ & 2 & 1 & 1 & 4 \\
$24.1-36$ & 3 & 1.5 & 1.5 & 6 \\
$36.1-48$ & 4 & 2 & 2 & 8 \\
$>48$ & 5 & 2.5 & 2.5 & 10 \\
\hline
\end{tabular}

in accordance with the standardized WHO protocol [11]. The treatment outcome was assessed on the basis of parasite clearance from the blood. Parasite clearance was defined as no parasite per 500 white blood cells by two continuous every 12-hour microscopy. The primary endpoint was the 28-day cure rate. Cure was defined as elimination of the symptoms and asexual blood stages of the malaria parasites that caused the patient to seek treatment. Fever clearance was defined as axillary temperatures $<37.1^{\circ}$ $C$ in duration of 24 hours [15].

\section{Classification standards for treatment outcome and sensitivity}

Treatment outcome was categorized based on the WHO definitions for early treatment failure (ETF), late clinical failure (LCF), late parasitological failure (LPF), and adequate clinical and parasitological response (ACPR). The ETF definition was to conform to any one of the criteria: (1) danger signs or severe malaria on day 1, 2 or 3 , in the presence of parasitaemia; (2) parasitaemia on day 2 higher than on day 0 , irrespective of axillary temperature; (3) parasitaemia on day 3 with axillary temperature $\geq 37.5^{\circ} \mathrm{C}$; and, (4) parasitaemia on day $3 \geq 25 \%$ of count on day 0 . The LCF definition was to satisfy any one of the criteria: (1) danger signs or severe malaria in the presence of parasitaemia on any day between day 4 and day 28 in patients who did not previously meet any of the criteria of ETF; and, (2) presence of parasitaemia on any day between day 4 and day 28 with axillary temperature $\geq 37.5^{\circ} \mathrm{C}$ in patients who did not previously meet any criteria of ETF. LPF definition was to satisfy presence of parasitaemia on any day between day 7 and day 28 with axillary temperature $<37.5^{\circ} \mathrm{C}$ in patients who did not previously meet any of the criteria of ETF or LCF. ACPR definition was to satisfy absence of parasitaemia on day 28 , irrespective of axillary temperature, in patients who did not previously meet any criteria of ETF, LCF or LPF [11].

Resistant responses (S, RI-RIII) were categorized according to the WHO criteria [13]. Susceptible parasites (S) were defined if asexual stage parasites had a $\geq 75 \%$ reduction within 48 hours after drug treatment and slides were negative for two consecutive days within seven days, and no recrudescence occurred within the 28-day follow-up period. RI definition was $75 \%$ reduction in parasitaemia within 48 hours after initiation of therapy and parasite clearance within seven days but recrudescence within 28 days; RII was $\geq 75 \%$ reduction of initial parasitaemia within 48 hours of drug treatment, but without parasite clearance within seven days; RIII was parasitaemia $\geq 25 \%$ of initial parasite count at 48 hours after initiation of therapy. Patients with resistant parasites (RI-RIII) were subsequently given a standard adult dosage of dihydroartemisinin-piperaquine tablets following the Ministry of Health recommended two-day 
treatment regimen (dihydroartemisinin $160 \mathrm{mg}$ and piperaquine $1280 \mathrm{mg} /$ day) [13].

\section{Statistical analysis}

The data were entered by Microsoft Office Excel 2007 and analysed by the Kaplan-Meier method $[11,16]$. The patients of loss to follow-up and withdrawal from the study were not involved into the analysis.

\section{Ethical approval}

According to the Helsinki Declaration, ethical approval for the study was granted by the Ethics Committee of Yunnan Institute of Parasitic Diseases, China. The purpose of the study was explained and then approval was sought from patients and their caretakers. Informed written consent was obtained from patients or from carers of child patients. All results were kept confidential and were unlinked to any identifying information.

\section{Results}

During 2008-2013, a total of 750 vivax malaria patients were recruited in the study, 14 withdrew, 133 were lost at follow-up, and 603 completed valid follow-up, of which 531 (88.1\%) were Burmese (Table 2). The fever clearance time (FCT) and asexual parasite clearance times (APCT) were, respectively, $22.2 \pm 10.2$ and $38.1 \pm$ 12.6 hours. The results showed that $594(98.5 \%)$ of the patients were ACPR, and nine (1.5\%) LCF, without ETF and LPF. Two LCF patients were observed in 2010, one in 2012 and six in 2013 (Table 3). All nine LCF patients responded well to the two-day treatment regimen of dihydroartemisinin-piperaquine tablets. The result of parasite sensitivity classification was the same as the treatment outcome. The number of susceptible parasites was 594 and the RI was nine (Table 4). The PCR identified nine $(1.5 \%)$ as recrudescence within day 28 , one recrudescence on day 14 and 28, respectively, in 2010; one on day 28 in 2012; one on day 14 and 21, respectively, and four others on day 28 in 2013 (Tables 3 and 4). The PCR did not identify any new infection of $P$. vivax. All nine patients displaying RI and LCF were from the neighbouring districts of Myanmar and no significant differences were identified between different classifications. 1.5\% (9/601) of LCF among imported cases from Myanmar including 531 Burmese and 70 Chinese who contracted malaria in Myanmar and come back to China for treatment versus $0 \%(0 / 2)$ locally acquired in China $\left(x^{2}=0.03, P=0.862\right) ; 1.7 \%(9 / 531)$ of LFC in Burmese and $0 \%(0 / 72)$ in Chinese $\left(x^{2}=0.35, P=0.552\right) .1 .0 \%(3 / 287)$ of LFC in adults ( $\geq 16$ years) versus $1.9 \%(6 / 316)$ in children $\left(x^{2}=0.28, P=0.598\right)$.

\section{Discussion}

WHO encourages countries in which $P$. vivax is endemic to carry out pilot studies to monitor CQ efficacy [11]. CQ is the first-line drug for treatment of blood stage infection of $P$. vivax and it has been used in treatment of vivax malaria for more than 50 years in China. The study monitored CQ therapeutic efficacy for uncomplicated vivax malaria on the China-Myanmar border from 2008 to 2013. The cumulative success rate for $P$. vivax treatment was $98.5 \%$. All nine treatment failures were LCF or RI resistance. Wei et al. carried out a study on CQ therapeutic efficacy for $P$. vivax treatment in China-Vietnam border from 1989 to 1998. They found P. vivax resistance to CQ in 1989 and identified three RI, eleven RII and three RIII in the ten

Table 2 Baseline characteristics of vivax malaria patients in Tengchong and Yingjiang counties, Yunnan, China

\begin{tabular}{|c|c|c|c|c|c|c|c|}
\hline & $2008(n=22)$ & $2009(n=9)$ & $2010(n=84)$ & $2011(n=43)$ & $2012(n=123)$ & $2013(n=322)$ & Total $(n=603)$ \\
\hline \multicolumn{8}{|l|}{ Sex } \\
\hline Male & $20(89.9 \%)$ & $8(88.9 \%)$ & $63(75.0 \%)$ & $28(65.1 \%)$ & $69(56.1 \%)$ & 165 (51.2\%) & $353(58.5 \%)$ \\
\hline Female & $2(9.1 \%)$ & $1(11.1 \%)$ & $21(25.0 \%)$ & $15(34.9 \%)$ & $54(43.9 \%)$ & $157(48.8 \%)$ & $250(41.5 \%)$ \\
\hline \multicolumn{8}{|l|}{ Nationality } \\
\hline Chinese & $22(100 \%)$ & 9 (100\%) & $18(21.4 \%)$ & $10(23.3 \%)$ & $8(6.5 \%)$ & $5(1.6 \%)$ & $72(11.9 \%)$ \\
\hline Burmese & 0 & 0 & $66(78.6 \%)$ & $33(76.7 \%)$ & 115 (93.5\%) & 317 (98.4\%) & $531(88.1 \%)$ \\
\hline \multicolumn{8}{|l|}{ Age (years) } \\
\hline Mean \pm SD & $20.2 \pm 1.4$ & $22.4 \pm 4.8$ & $28.4 \pm 8.2$ & $33.5 \pm 6.4$ & $26.2 \pm 10.2$ & $18.3 \pm 9.6$ & $25.2 \pm 6.8$ \\
\hline Range & $23-50$ & $31-53$ & $2-59$ & $2-60$ & $2-59$ & $1-60$ & $1-60$ \\
\hline \multicolumn{8}{|c|}{ Body temperature $\left({ }^{\circ} \mathrm{C}\right)$} \\
\hline Mean \pm SD & $38.1 \pm 0.4$ & $38.1 \pm 0.8$ & $38.5 \pm 0.5$ & $39.3 \pm 0.5$ & $38.8 \pm 0.6$ & $38.2 \pm 0.8$ & $38.5 \pm 0.7$ \\
\hline Rang & $36.3-39.5$ & $37.0-39.4$ & $36.8-40$ & $37.1-41.0$ & $36.6-40.0$ & $36.5-40.8$ & \\
\hline \multicolumn{8}{|c|}{ Parasite count (per $\mu \mathrm{l}$ ) } \\
\hline Geometric mean & 7,621 & 6,327 & 8,675 & 6,732 & 8,976 & 8,156 & 7,881 \\
\hline Range (per $\mu l$ ) & $556-25,606$ & $1,582-18,334$ & $521-102,500$ & $663-99,130$ & $546-111,960$ & $586-97,846$ & $521-111,960$ \\
\hline
\end{tabular}


Table 3 Treatment responses of vivax malaria patients in Tengchong and Yingjiang counties, Yunnan, China

\begin{tabular}{llllllll}
\hline & $\mathbf{2 0 0 8}(\mathbf{n}=\mathbf{2 2})$ & $\mathbf{2 0 0 9}(\mathbf{n}=\mathbf{9})$ & $\mathbf{2 0 1 0}(\mathbf{n}=\mathbf{8 4})$ & $\mathbf{2 0 1 1}(\mathbf{n}=\mathbf{4 3})$ & $\mathbf{2 0 1 2}(\mathbf{n}=\mathbf{1 2 3})$ & $\mathbf{2 0 1 3}(\mathbf{n}=\mathbf{3 2 2})$ & Total (n= 603) \\
\hline ETF & 0 & 0 & 0 & 0 & 0 & 0 & 0 \\
LCF & 0 & 0 & $2(2.4 \%)$ & 0 & $1(0.8 \%)$ & $6(1.9 \%)$ & $9(1.5 \%)$ \\
LPF & 0 & 0 & 0 & 0 & 0 & 0 & 0 \\
ACPR & $22(100 \%)$ & $9(100 \%)$ & $82(97.6 \%)$ & $43(100 \%)$ & $122(99.2 \%)$ & $316(98.1 \%)$ & $594(98.5 \%)$ \\
\hline
\end{tabular}

Note: $\mathrm{ETF}=$ early treatment failure, $\mathrm{LCF}=$ late clinical failure, $\mathrm{LPF}=$ late parasitological failure, and $\mathrm{ACPR}=$ adequate clinical and parasitological response.

years. The cumulative failure rate (CFR) was $37.1 \%(17 /$ 47) [17]. Yang et al. observed 100 vivax malaria patients in central Yunnan Province in 1995, two RI and two RII were identified and the CFR was $4.0 \%(4 / 100)$ [18]. In the two studies, patients with RI-RII $P$. vivax were subsequently given a standard dosage of intramuscular artemether injections following the Ministry of Health's recommended fiveday treatment regimen $(160 \mathrm{mg}$ on day 0 and $80 \mathrm{mg}$ daily for four days) $[17,18]$. Liang et al. reported one RI and CFR $1.9 \%(1 / 54)$ on the same China-Myanmar border in 2008. They treated the CQ-resistant parasites with the two-day treatment regimen of dihydroartemisinin-piperaquine tablets [19]. Six CQ-resistant isolates were identified through in vitro microtest in central China in 2005 [20]. The $P$. vivax resistance to CQ was also found in Myanmar in 1990s [21-23]. Guthmann et al. reported that 34.1\% (80/ 235) of vivax malaria patients with CQ treatment had recurrent parasitaemia and were considered treatment failures in southern Myanmar between December 2002 and April 2003 [24]. In other parts of the world, CQ resistance in $P$. vivax has been documented across the Indonesian archipelago [25], South Korea [26], Turkey [27], the Horn of Africa [28], and South America [29]. These studies showed that there are increasing worldwide reports of CQ resistance in $P$. vivax, however they did not indicate significant change of CQ resistance $[9,30]$.

The pace of CQ resistance development in P. vivax depends on numerous factors, such as drug selective pressure, endemicity, host immunity, and varies from region to region. CQ was used for clinical and prophylactic treatment of both $P$. vivax and Plasmodium falciparum in China in 1980s. The drug policy was changed in response to $P$. falciparum drug resistance, and then CQ was used only for vivax malaria treatment since 1990s. This may be one of reasons that the CFR (1.5\%) of the study is much lower than the CFR (37.1\%) between 1989 and 1998 in
Yunnan Province, China [17,18]. One of Wei et al. and Yang et al. studies' technical limitations was not to use PCR to distinguish between re-infection and recrudescence to confirm the Plasmodium species or to detect mixed infection, and to exclude microscopic misidentification of $P$ falciparum as $P$ vivax when $P$ falciparum prevalence was higher during 1989-98 and young ring stages difficult to distinguish. These might have increased their CFRs. Yunnan is a large province, with Wei et al. doing their study on the China-Vietnam border and Yang et al. in central Yunnan, whereas the current and other studies reported were done on the China-Myanmar border. Geographic differences may be one of explanations for reported differences too. Tengchong and Yingiiang are in a malaria-eliminating phase, so PCR did not identify any new infection within day 28. LCF of the study was observed in 2010, 2012 and 2013 and all nine LCF patients were imported from neighbouring areas of Myanmar. This shows that CQ resistance has not increased in recent years in Yunnan Province. CFR $1.9 \%(1 / 54)$ in 2008 [19], 2.4\% (2/84) in 2010, $0.8 \%(1 / 123)$ in 2012 and $1.9(6 / 322)$ in 2013 showed that CQ resistance did not significantly change $\left(x^{2}=0.86, P=0.834\right)$ along the China-Myanmar border.

Despite PCR being used to distinguish between reinfection and recrudescence, to confirm the Plasmodium species or to detect mixed infection in the study, $P$. vivax can relapse from long-lasting liver stages [11,23]. It is one limitation for the study and remains a challenge for monitoring in vivo P. vivax drug resistance. However, most of $P$. vivax in tropical areas is usually an early relapsing strain despite the late relapsing strain (temperate zone strains) exists in some high altitude (around 3,000 metres above the sea level) areas in Yunnan. The study was carried out in tropical areas and most of malaria patients were infected in Myanmar, so most of them should be tropical strain or early relapsing strain. This would reduce the PCR

Table 4 Clinical resistance of Plasmodium vivax in Tengchong and Yingjiang counties, Yunnan, China

\begin{tabular}{llllllll}
\hline & $\mathbf{2 0 0 8 ( n = 2 2 )}$ & $\mathbf{2 0 0 9}(\mathbf{n = 9 )}$ & $\mathbf{2 0 1 0}(\mathbf{n}=\mathbf{8 4})$ & $\mathbf{2 0 1 1}(\mathbf{n}=\mathbf{4 3})$ & $\mathbf{2 0 1 2}(\mathbf{n}=\mathbf{1 2 3})$ & $\mathbf{2 0 1 3}(\mathbf{n}=\mathbf{3 2 2})$ & Total $(\mathbf{n}=\mathbf{6 0 3})$ \\
\hline S & $22(100 \%)$ & $9(100 \%)$ & $82(97.6 \%)$ & $43(100 \%)$ & $122(99.2 \%)$ & $316(96.1 \%)$ & $594(98.5 \%)$ \\
RI & 0 & 0 & $2(2.4 \%)$ & 0 & $1(0.8 \%)$ & $6(3.9 \%)$ & $9(1.5 \%)$ \\
R II & 0 & 0 & 0 & 0 & 0 & 0 & 0 \\
R III & 0 & 0 & 0 & 0 & 0 & 0 & 0 \\
\hline
\end{tabular}

Note: $\mathrm{S}$ = susceptible, $\mathrm{RI}$ = resistant level I, RII = resistant level II, RIII = resistant level III. 
limitation in distinguishing recrudescence from relapse. Another limitation of the study is the change of surveillance sites, in Tengchong in 2008-2009, in both Tengchong and Yingjiang in 2010-2011, and in Yingjiang alone in 2012-2013. However, both monitoring sites are in the same section of the China-Myanmar border, so the study results indicate $P$. vivax sensitivity to CQ in the target region. The third limitation is that the number of valid study cases was only 22 in 2008, nine in 2009 and 43 in 2011, which may be the reason that CQ resistance was not found in those three years. The forth limitation is that children $<1$ year and over 60 years old persons were excluded from the study, so the results may not be applicable to these two age groups.

\section{Conclusion}

In terms of efficacy, CQ is still effective for vivax malaria treatment. Plasmodium vivax CQ sensitivity has not significantly changed on the China-Myanmar border of Yunnan Province, China.

\section{Competing interests}

The authors declare that they have no competing interests.

\section{Authors' contributions}

$L-H T, H-L Y, H L, F H$, and $M L$ designed the study and developed the protocol. J-W X and $\mathrm{HL}$ analysed and interpreted the data. HL organized and supervised the study in field. L-HT, H-L Y, FH, and ML monitored the study in general. HL, $X-L L, J-Z W, C-F L, H-Y W, R-H N, X-R G$, and $Y-X L$ conducted the study in field, and entered the data. J-WX and $\mathrm{HL}$ wrote the first draft of the paper. All authors read and approved the final manuscript.

\section{Acknowledgements}

This study was supported by The WHO Mekong Malaria Programme (WP/10/ MVP/005837). We thank all participants for their contribution of time and patience in the study. We also thank staff of Yunnan Institute of Parasitic Diseases (YIPD) for logistic support, and clinical and laboratory staff of Tengchong and Yangjiang County Center for Disease Control and prevention for their hard work. The opinions expressed are those of the authors and do not necessarily reflect those of WHO and YIPD.

\section{Author details}

${ }^{1}$ Yunnan Institute of Parasitic Diseases, Puer 665000, China. ${ }^{2}$ National Institute of Parasitic Diseases, Chinese Center for Disease Control and Prevention, Shanghai 200025, China. ${ }^{3}$ Tengchong County Center for Disease Control and Prevention, Tengchong, China. ${ }^{4}$ Yangjiang County Center for Disease Control and Prevention, Tengchong, China.

Received: 26 June 2014 Accepted: 12 September 2014 Published: 15 September 2014

\section{References}

1. WHO: World Malaria Report 2011. Geneva: World Health Organization; 2011.

2. Ministry of Health, People's Republic of China: From Malaria Control to Elimination: a Revised National Malaria Strategy 2010-2015. Beijing: Ministry of Health; 2009:2-4.

3. Ministry of Health, People's Republic of China: Malaria Elimination Action Plan 2010-2020(in Chinese). Beijing: Ministry of Health; 2010:1-2.

4. Cotter C, Sturrock HJW, Hsiang MS, Liu J, Phillips AA, Hwang J, Gueye CS, Fullman $N$, Gosling RD, Feachem RGA: The changing epidemiology of malaria elimination: new strategies for new challenges. Lancet 2013, 382:900-911.

5. Petersen I, Eastman R, Lanzer M: Drug-resistant malaria: molecular mechanisms and implications for public health. FEBS Lett 2011, 585:1551-1562.

6. Cui L, Yan G, Sattabongkotc J, Chen D, Cao Y, Fan Q, Parkera D, Sirichaisinthopg J, Su XZ, Yang H, Yang Z, Wang B, Zhou G: Challenges and prospects for malaria elimination in the Greater Mekong. Acta Trop 2012, 121:240-245.

7. Price RN, Tjitra E, Guerra CA, Yeung S, White NJ, Anstey NM: Vivax malaria: neglected and not benign. Am J Trop Med Hyg 2007, 77:79-87.

8. Price RN, Douglas NM, Anstey NM: New developments in Plasmodium vivax malaria: severe disease and the rise of chloroquine resistance. Curr Opin Infect Dis 2009, 22:430-435.

9. Rieckmann KH, Davis DR, Hutton DC: Plasmodium vivax resistance to chloroquine? Lancet 1989, 2:1183-1184.

10. Phyo AP, Lwin KM, Price RN, Ashley EA, Russell B, Sriprawat K, Lindegardh N, Singhasivanon P, White NJ, Nosten F: Dihydroartemisinin-piperaquine versus chloroquine in the treatment of Plasmodium vivax malaria in Thailand: a randomized controlled trial. Clin Infect Dis 2011, 53:977-984.

11. World Health Organization: Method for Surveillance of Antimalarial Drug Efficacy. Geneva: World Health Organization; 2009:12-82. http://www.who. int/malaria/resistance.

12. Bureau for Disease control and prevention, Ministry of Health, People's Republic of China: Malaria Control Manual (in Chinese). Beijing: People's Health Publishing Company; 2007:33-42.

13. Yang $\mathrm{HL}$, Yang $Y$, Yang PF: Monitoring Plasmodium falciparum chloroquine resistance in Yunnan Province, China, 1981-2006. Acta Trop 2008, 108:44-49.

14. Huang $T$, Wang $X$, Li X, Huang $Y$, Zeng C, Zhang S, Fu W, Zhang Z, Zhang G: Studies on identification of circumsporozoite protein genotyping of Plasmodium vivax] (in Chinese). Chin J Parasitol Parasit Dis 2000, 18:272-276.

15. Organization WH: Guidelines for the Treatment of Malaria. Geneva: World Health Organization; 2006:1-66.

16. Stepniewska K, White NJ: Some considerations in the design and interpretation of antimalarial drug trials in uncomplicated falciparum malaria. Malar J 2006, 5:127.

17. Wei D, Li GL, Huang YS, Dai CF, Chen GL: The analysis of clinical characteristics for 47 patient cases of chloroquine-resistant Plasmodium vivax (in Chinese). Southwestern Defence Med 2000, 10:86-87.

18. Yang XM, Yang MQ, Huang JW, Zhao FX: The clinical study on Plasmodium vivax chloroquine sensitivity (in Chinese). Chinese J Parasitic Dis Control 1996, 9:226-227.

19. Liang GL, Su XD, Wang J, Zhang ZX: Evaluation of the sensitivity of vivax malaria to chloroquine in the City of Laza, Myanmar (in Chinese). J Pathogen Biol 2010, 5:362-372.

20. Lu F, Gao Q, Xia H, Tao Z, Cao J, Gu Y, Zhou H, Jin X, Udomsangpetch R, Sattabongkot J: In vitro sensitivity test of Plasmodium vivax to chloroquine in center part of China (in Chinese). Chin J Schisto Control 2006, 18:265-267.

21. Myat Phone K, Myint O, Myint L, Thaw Z, Kyin Hla A, Nwe Nwe Y: Emergence of chloroquine-resistant Plasmodium vivax in Myanmar (Burma). Trans R Soc Trop Med Hyg 1993, 87:687.

22. Marlar T, Myat Phone K, Aye Yu S, Khaing Khaing G, Ma S, Myint $O$ : Development of resistance to chloroquine by Plasmodium vivax in Myanmar. Trans R Soc Trop Med Hyg 1995, 89:307-308.

23. Barnadas C, Kent K, Timinao1 L, Iga1 J, Gray L, Siba1 P, Mueller I, Thomas P, Zimmerman P: A new high-throughput method for simultaneous detection of drug resistance associated mutations in Plasmodium vivax dhfr, dhps and mdr1 genes. Malar J 2011, 10:282.

24. Guthmann JP, Pittet A, Lesage A: Plasmodium vivax resistance to chloroquine in Dawei, southern Myanmar. Trop Med Int Health 2008, 13:91-98.

25. Baird JK: Chloroquine resistance in Plasmodium vivax. Antimicrob Agents Chemother 2004, 48:4075-4083.

26. Lee KS, Kim TH, Kim ES: Chloroquine-resistant Plasmodium vivax in the Republic of Korea. Am J Trop Med Hyg 2009, 80:215-217.

27. Kurcer MA, Simsek Z, Zeyrek FY: Efficacy of chloroquine in the treatment of Plasmodium vivax malaria in Turkey. Ann Trop Med Parasitol 2004, 98:447-451.

28. Teka H, Petros B, Yamuah L: Chloroquine-resistant Plasmodium vivax malaria in Debre Zeit, Ethiopia. Malar J 2008, 7:220.

29. Santana Filho FS, Arcanjo AR, Chehuan YM: Chloroquine resistant Plasmodium vivax, Brazilian Amazon. Emerg Infect Dis 2007, 13:1125-1126.

30. Douglas N, Nicholas N, Angus B, Nosten F, Price R: Artemisinin combination therapy for vivax malaria. Lancet Infect Dis 2010, 10:405-416.

doi:10.1186/1475-2875-13-364

Cite this article as: Liu et al:: Monitoring Plasmodium vivax chloroquine sensitivity along China-Myanmar border of Yunnan Province, China during 2008-2013. Malaria Journal 2014 13:364. 